\title{
In-Flight Self-Alignment Method Aided by Geomagnetism for Moving Basement of Guided Munitions
}

\author{
Shuang-biao Zhang, ${ }^{1,2}$ Xing-cheng Li, ${ }^{1,2}$ and Zhong Su${ }^{3}$ \\ ${ }^{1}$ Beijing Institute of Technology, Beijing 100081, China \\ ${ }^{2}$ Key Laboratory of Dynamics and Control of Flight Vehicle, Ministry of Education, Beijing 100081, China \\ ${ }^{3}$ Beijing Key Laboratory of High Dynamic Navigation Technology, University of Beijing Information Science \& Technology, \\ Beijing 100101, China \\ Correspondence should be addressed to Xing-cheng Li; xingchli@bit.edu.cn
}

Received 2 March 2015; Revised 4 May 2015; Accepted 11 May 2015

Academic Editor: Kalyana C. Veluvolu

Copyright (C) 2015 Shuang-biao Zhang et al. This is an open access article distributed under the Creative Commons Attribution License, which permits unrestricted use, distribution, and reproduction in any medium, provided the original work is properly cited.

\begin{abstract}
Due to power-after-launch mode of guided munitions of high rolling speed, initial attitude of munitions cannot be determined accurately, and this makes it difficult for navigation and control system to work effectively and validly. An in-flight self-alignment method aided by geomagnetism that includes a fast in-flight coarse alignment method and an in-flight alignment model based on Kalman theory is proposed in this paper. Firstly a fast in-flight coarse alignment method is developed by using gyros, magnetic sensors, and trajectory angles. Then, an in-flight alignment model is derived by investigation of the measurement errors and attitude errors, which regards attitude errors as state variables and geomagnetic components in navigation frame as observed variables. Finally, fight data of a spinning projectile is used to verify the performance of the in-flight self-alignment method. The satisfying results show that (1) the precision of coarse alignment can attain below $5^{\circ}$; (2) the attitude errors by in-flight alignment model converge to $24^{\prime}$ at early of the latter half of the flight; (3) the in-flight alignment model based on Kalman theory has better adaptability, and show satisfying performance.
\end{abstract}

\section{Introduction}

Guided munitions improved from conventional artillery shells attract more global attention for their outstanding advantages such as low cost, high battle effectiveness, and high stability. In practical applications, the power-beforelaunch mode is chosen for guided munitions. But this mode frequently induces the electrical circuits systems fixed in guided munitions to shock fiercely, and the electrical circuits systems are damaged to be invalid by momentary impact force. Thus, a more appropriate power-after-launch mode, as an ideal power mode, is raised to ensure the safety of the electrical circuits systems. The only drawback of this power mode is that initial attitude cannot be obtained accurately, and this makes it hard for navigation and control system to work effectively and validly. Therefore, to guarantee implement of guidance, it is imperative for the power-after-launch mode of guided munitions to exploit an effective approach of initial alignment.

Up to now, many achievements on in-flight alignment and alignment of moving basement are undergoing by using Global Position System (GPS), due to the fact that GPS can provide accurate position for carriers in need. A strapdown inertial navigation system (SINS) in-flight alignment algorithm proposed by Mei decomposes the attitude quaternion into three parts: inertial rate, separate earth motion, and a constant quaternion. The first two parts are solved based on Microelectromechanical Systems (MEMS) gyros' measurement and GPS position information, respectively, and the constant quaternion is solved by using request method [1]. A coarse alignment method is presented by Qian et al. to acquire the initial position, velocity, and attitude information with the help of GPS. The method could be implemented in airplanes of high speed and high dynamic flight without any restrictive 
condition of dynamic process and flight stability [2]. A robust filter and various error models for SINS in-flight alignment are presented by $\mathrm{Yu}$ and Lee to deal with the uncertainty by linearization of the system that degrades the performance of the filter. This method shows fine performance of estimation if enough time is allowed [3]. A $H_{\infty}$ filter designed by Pei et al. can analyze the uncertainty of measurement noise and realize in-flight alignment of aircrafts [4]. A technique for constructing the initial alignment system for unmanned aerial vehicles is proposed by Veremeenko and Savel'Ev to improve the accuracy of alignment and reduce the alignment time [5]. An estimation algorithm is proposed by She et al. to calculate initial roll angle of guided munitions under small angle of attack with the help of GPS [6]. An adaptive filtering algorithm of an extended Kalman filter (EKF) combined with innovationbased adaptive estimation is proposed by Fang and Yang to realize initial alignment, and the algorithm introduces the calculated innovation covariance into the computation of the filter gain matrix directly [7]. Meanwhile, an estimation method is presented by Silson for fast initial coarse alignment of a ship's strapdown inertial attitude reference system using only inertial measurement unit (IMU) measurements for quasi-static alignment and IMU measurements with GPS aiding for moving-base alignment [8]. An improved selfalignment scheme for SINS based on the EKF and augmented measurements is proposed by Acharya et al. Monte Carlo simulations show that the method provides fine azimuth alignment and improves rate of convergence of azimuth attitude error and reduction in sensitivity to gyro biases [9]. A novel method using the GPS measurements is investigated by $\mathrm{Ma}$ et al. to help to solve coarse alignment. Specific vectors are constructed with GPS measurements and used for attitude matrix computation [10]. In addition, a nonlinear approach for the in-motion alignment problem of inertial navigation system using Doppler speed measurements with unknown initial vehicle is proposed by Bimal Raj and Joshi, and an unscented Kalman filter for alignment shows better performance than that of extended Kalman filter [11]. Body frame velocity from Doppler Velocity $\log (\mathrm{DVL})$ is used by $\mathrm{Li}$ et al. to construct the vector observations for initial alignment of underwater vehicles [12].

At our best knowledge, applications of satellite navigation technique are dependent on authorization, and it is easy to disturb signal of satellites by other strong signals on purpose. Moreover, GPS and Doppler technique are suitable for low dynamical carrier, especially for the carrier with low rolling speed or without rolling. In other words, there is no valid way to implement initial alignment of guided munitions with high spinning speed and power-after-launch mode.

In this paper, we represent an in-flight self-alignment method of moving basement aided by geomagnetism, which includes a fast in-flight coarse alignment method and an inflight alignment model based on Kalman theory. The rest of this paper is organized as follows. In the second section, we exploit a fast in-flight coarse alignment method by using gyros, magnetic sensors, and trajectory angle. The third section describes an in-flight alignment model by using Kalman theory to estimates the attitude angles. The case study is given in the fourth section. Finally, we conclude this paper in the fifth section.

\section{A Fast In-Flight Coarse Alignment Method}

In general, the initial alignment consists of two stages, the coarse alignment and the fine alignment. The coarse alignment is the basic step to supply initial attitude for the fine alignment. This process is crucial but difficult to estimate attitude angle of munitions with high spinning speed and powerafter-launch mode. Thus, in this section, we investigate a fast in-flight coarse alignment method to calculate initial attitude by analyzing the angular kinematic model and attitude resolving algorithm of magnetic sensors.

For convenience of investigation, we choose east-northup frame as navigation frame, and the angular kinematic models are described as [13]

$$
\left[\begin{array}{c}
\dot{\gamma} \\
\dot{\vartheta} \\
\dot{\psi}
\end{array}\right]=\left[\begin{array}{ccc}
1 & -\sin \gamma \tan \vartheta & -\cos \gamma \tan \vartheta \\
0 & \cos \gamma & -\sin \gamma \\
0 & \frac{\sin \gamma}{\cos \vartheta} & \frac{\cos \gamma}{\cos \vartheta}
\end{array}\right]\left[\begin{array}{l}
\omega_{b x} \\
\omega_{b y} \\
\omega_{b z}
\end{array}\right],
$$

where $\gamma$ is the roll angle, $\vartheta$ is pitch angle, $\psi$ is yaw angle, $\dot{\gamma}, \dot{\vartheta}$, and $\dot{\psi}$ are the corresponding angle changing rates, and $\omega_{b x}$, $\omega_{b y}$, and $\omega_{b z}$ are measurement data of MEMS gyros.

By transforming (1), the pitch angle can be formulated by

$$
\vartheta= \pm \arccos \sqrt{\frac{\omega_{b y}^{2}+\omega_{b z}^{2}-\dot{\vartheta}^{2}}{\dot{\psi}^{2}}} .
$$

From (2), it is seen that the symbol of pitch angle is positive in the rising stage of guided munitions, and it is opposite in the falling stage. To implement coarse alignment and ensure enough time for fine alignment, the pitch angle must be determined in the rising stage. So its symbol of pitch angle is positive.

Assuming aerodynamic force and gravity that guided munitions suffer vary slightly, the influence of coning motion is ignored. The pitch angle, yaw angle, and their corresponding changing rates can be regarded as long-periodic variables. Meanwhile, the changing rates of pitch angle and yaw angle vary slowly and even keep invariant during very short time. We can formulate $\dot{\vartheta}$ and $\dot{\psi}$ by the ideal ballistic trajectory angles, inclination angle $\theta$, and heading angle $\psi_{v}$ :

$$
\begin{aligned}
& \dot{\vartheta} \approx \dot{\theta} \approx \frac{\theta(t+\Delta t)-\theta(t)}{\Delta t}, \\
& \dot{\psi} \approx \dot{\psi}_{v} \approx \frac{\psi_{v}(t+\Delta t)-\psi_{v}(t)}{\Delta t},
\end{aligned}
$$


where $\Delta t$ is time interval. Equation (3) can be used to calculate data on line. If some calculations need to be done offline, $\theta$ and $\psi_{v}$ can be calculated by

$$
\begin{aligned}
\theta & =\arctan \frac{v_{y}}{\sqrt{v_{x}^{2}+v_{z}^{2}}}, \\
\psi_{v} & =-\arctan \frac{v_{z}}{v_{x}},
\end{aligned}
$$

where $v_{x}, v_{y}$, and $v_{z}$ are components of velocity in navigation frame, and they can be calculated as

$$
\begin{aligned}
& v_{x}=\frac{x(t+\Delta t)-x(t)}{\Delta t}, \\
& v_{y}=\frac{y(t+\Delta t)-y(t)}{\Delta t}, \\
& v_{z}=\frac{z(t+\Delta t)-z(t)}{\Delta t},
\end{aligned}
$$

where $x, y$, and $z$ are components of location. In fact, the real ballistic trajectory angles are similar to the ideal ones.

Due to low cost of the MEMS sensors, they are familiar in navigation and control system of guided munitions. However, narrow measurement range and poor accuracy of existing MEMS gyros cannot meet measurement requirement of guided munitions with high rolling speed of $x$-axis. To solve the problem of calculation of roll angle, we make use of MEMS magnetic sensors that are fixed in guided munitions to help navigation computer calculate attitude.

The attitude algorithm of geomagnetic technology has been investigated for several years. It has high level of resolving precision and satisfying instantaneity, so we can resolve attitude in real-time. The formulations of yaw angle and roll angle are described as [14]

$$
\psi=D+\arccos \frac{m_{b x} / B+\sin \vartheta \sin I}{\cos \vartheta \cos I}
$$

$$
\left[\begin{array}{c}
\dot{\gamma}+\delta \dot{\gamma} \\
\dot{\vartheta}+\delta \dot{\vartheta} \\
\dot{\psi}+\delta \dot{\psi}
\end{array}\right]=\left[\begin{array}{cc}
1 & -\sin (\gamma+\delta \gamma) \tan (\vartheta+\delta \vartheta) \\
0 & \cos (\gamma+\delta \gamma) \\
0 & \frac{\sin (\gamma+\delta \gamma)}{\cos (\vartheta+\delta \vartheta)}
\end{array}\right.
$$

where $\delta \gamma, \delta \vartheta$, and $\delta \psi$ are attitude errors, $\delta \dot{\gamma}, \delta \dot{\vartheta}$, and $\delta \dot{\psi}$ are changing rates errors of attitude, and $\delta \omega_{b x}, \delta \omega_{b y}$, and $\delta \omega_{b z}$ are measurement errors of gyros.

As long as the attitude errors are enough small, which are assumed less than five degree, the trigonometric functions in (7) can be simplified by using $\sin (\delta x) \approx \delta x$ and $\cos (\delta x) \approx 1$ :

$$
\begin{aligned}
& \tan (\vartheta+\delta \vartheta) \approx \tan \vartheta+\left(1+\tan ^{2} \vartheta\right) \delta \vartheta, \\
& \cos (\gamma+\delta \gamma) \approx \cos \gamma-\delta \gamma \sin \gamma, \\
& \sin (\gamma+\delta \gamma) \approx \sin \gamma+\delta \gamma \cos \gamma,
\end{aligned}
$$

$$
\begin{aligned}
\gamma= & \arctan \frac{\cos I \sin (\psi-D)}{\cos (\psi-D) \cos I \sin \vartheta+\sin I \cos \vartheta} \\
& +\arctan \frac{m_{b y}}{m_{b z}},
\end{aligned}
$$

where $I$ and $D$ are, respectively, inclination angle and declination angle of geomagnetic vector and $m_{b x}, m_{b y}$, and $m_{b z}$ are measurement values of MEMS magnetic sensors in body frame. From (6), we can see that the yaw angle is related to measurement value of the longwise magnetic sensor, and the roll angle is related to lateral measurement values. By combining (2) and (6), the initial attitude can be determined, and the coarse alignment can be realized.

Generally speaking, the firing range of munitions is about several kilometers or scores of kilometers, and the geomagnetic vector changes slightly. Thus, the geomagnetic information can be stored in the navigation computer before launching. It is convenient to extract geomagnetic information when guided munitions are in-flight.

\section{An In-Flight Alignment Model Based on Kalman Theory}

Based on the coarse alignment, it is ready to make fine alignment for navigation and control system. In this section, we develop an in-flight alignment model based on Kalman theory to estimate angle errors and achieve fine alignment.

The measurement errors of MEMS gyros sensing lateral control channels exist all the time because of their poor measurement precision. So the measurement values of gyros contain actual angular speed and error information, and the attitude resolved by (1) also contains actual attitude and error information. Thus, the actual formulations of (1) can be improved as

$$
\left.\begin{array}{c}
-\cos (\gamma+\delta \gamma) \tan (\vartheta+\delta \vartheta) \\
-\sin (\gamma+\delta \gamma) \\
\frac{\cos (\gamma+\delta \gamma)}{\cos (\vartheta+\delta \vartheta)}
\end{array}\right]\left[\begin{array}{l}
\omega_{b x}+\delta \omega_{b x} \\
\omega_{b y}+\delta \omega_{b y} \\
\omega_{b z}+\delta \omega_{b z}
\end{array}\right],
$$

$$
\frac{1}{\cos (\vartheta+\delta \vartheta)} \approx \frac{\cos \delta \vartheta}{\cos \vartheta}-\frac{\sin \delta \vartheta}{\sin \vartheta} \approx \frac{1}{\cos \vartheta}-\frac{\delta \vartheta}{\sin \vartheta}
$$

Thus, we can simplify (7) by substituting (8) into (7). Meanwhile, comparing (1) with simplified formulation of (7), the differential equations of attitude errors can be obtained:

$$
\begin{aligned}
\delta \dot{\gamma}= & \delta \gamma\left(\omega_{b y} \tan \vartheta \sin \gamma-\omega_{b z} \tan \vartheta \cos \gamma\right) \\
& -\delta \vartheta\left(1+\tan ^{2} \vartheta\right)\left(\omega_{b y} \cos \gamma+\omega_{b z} \sin \gamma\right) \\
& +\Delta_{x},
\end{aligned}
$$




$$
\begin{aligned}
\delta \dot{\vartheta}= & -\delta \gamma\left(\omega_{b y} \sin \gamma+\omega_{b z} \cos \gamma\right)+\Delta_{y}, \\
\delta \dot{\psi}= & \delta \vartheta\left(-\frac{\omega_{b y} \sin \gamma+\omega_{b z} \cos \gamma}{\sin \vartheta}\right) \\
& +\delta \gamma\left(\frac{\omega_{b y} \cos \gamma-\omega_{b z} \sin \gamma}{\cos \vartheta}\right)+\Delta_{z},
\end{aligned}
$$

where $\Delta_{x}, \Delta_{y}$, and $\Delta_{z}$ are drift errors of equivalent gyros. From (11), it is seen that yaw angle error may be singular if pitch angle is zero.

By dealing with (9)-(11), the state formulation based on Kalman theory can be described as

$$
\dot{X}=F X+G \Delta,
$$

where $F$ is state transition matrix and defined as

$$
F=\left[\begin{array}{lll}
F_{11} & F_{12} & F_{13} \\
F_{21} & F_{22} & F_{23} \\
F_{31} & F_{32} & F_{33}
\end{array}\right]
$$

where

$$
\begin{aligned}
& F_{11}=\omega_{b y} \tan \vartheta \sin \gamma-\omega_{b z} \tan \vartheta \cos \gamma, \\
& F_{12}=\left(1+\tan ^{2} \vartheta\right)\left(\omega_{b y} \cos \gamma+\omega_{b z} \sin \gamma\right), \\
& F_{13}=0, \\
& F_{21}=\omega_{b y} \sin \gamma+\omega_{b z} \cos \gamma, \\
& F_{22}=0, \\
& F_{23}=0, \\
& F_{31}=\frac{\omega_{b y} \cos \gamma-\omega_{b z} \sin \gamma}{\cos \vartheta}, \\
& F_{32}=-\frac{\omega_{b y} \sin \gamma+\omega_{b z} \cos \gamma}{\sin \vartheta}, \\
& F_{33}=0 .
\end{aligned}
$$

$X$ is state variable matrix, $X=\left[\begin{array}{lll}\Delta \gamma & \Delta \vartheta & \Delta \psi\end{array}\right]^{T}$. G is system noise drive matrix, $G=\left[\begin{array}{lll}1 & 0 & 0 \\ 0 & 1 & 0 \\ 0 & 0 & 1\end{array}\right] . \Delta$ is system noise matrix, $\Delta=\left[\begin{array}{lll}\Delta_{x} & \Delta_{y} & \Delta_{z}\end{array}\right]^{T}$.

The main frame of mind to establish observing matrix is as follows. We transform the measurement values of MEMS magnetic sensors fixed in body frame to navigation frame and transform the geomagnetic vector to navigation frame. Then we regard the discrepancy of comparison between the two parts as the observed quantities to estimate the state values. Considering about attitude errors, the actual transform matrix contains the errors matrix. The observed equation can be formulated by

$$
C_{m}^{n}\left[\begin{array}{l}
0 \\
B \\
0
\end{array}\right]=\left(\widetilde{C}_{b}^{n}-\delta C_{b}^{n}\right)\left[\begin{array}{l}
m_{b x} \\
m_{b y} \\
m_{b z}
\end{array}\right],
$$

where $\delta C_{b}^{n}$ is error matrix, and it can be described as

$$
\delta C_{b}^{n}=\left[\begin{array}{lll}
\delta C_{11} & \delta C_{12} & \delta C_{13} \\
\delta C_{21} & \delta C_{22} & \delta C_{23} \\
\delta C_{31} & \delta C_{32} & \delta C_{33}
\end{array}\right]
$$

Each element in $\delta C_{b}^{n}$ is described as

$$
\begin{aligned}
& \delta C_{11}=\cos \vartheta \cos \psi \Delta \psi-\sin \vartheta \Delta \vartheta \sin \psi, \\
& \delta C_{12}=-\left(\begin{array}{c}
\sin \gamma \sin \vartheta \cos \psi \Delta \psi \\
+\sin \gamma \cos \vartheta \Delta \vartheta \sin \psi \\
+\cos \gamma \Delta \gamma \sin \vartheta \sin \psi
\end{array}\right) \\
& +(\cos \gamma \sin \psi \Delta \psi+\sin \gamma \Delta \gamma \cos \psi), \\
& \delta C_{13}=-\left(\begin{array}{c}
\sin \gamma \Delta \gamma \sin \vartheta \sin \psi \\
+\cos \gamma \cos \vartheta \Delta \vartheta \sin \psi \\
+\cos \gamma \sin \vartheta \cos \psi \Delta \psi
\end{array}\right) \\
& +(\cos \gamma \Delta \gamma \cos \psi-\sin \gamma \cos \psi \Delta \psi) \text {, } \\
& \delta C_{21}=-\cos \vartheta \sin \psi \Delta \psi-\sin \vartheta \cos \psi \Delta \vartheta \text {, } \\
& \delta C_{22}=-\left(\begin{array}{c}
\sin \gamma \sin \vartheta \sin \psi \Delta \psi \\
+\sin \gamma \cos \vartheta \Delta \vartheta \cos \psi \\
+\cos \gamma \Delta \gamma \sin \vartheta \sin \psi
\end{array}\right) \\
& +(\cos \gamma \cos \psi \Delta \psi-\sin \gamma \Delta \gamma \sin \psi), \\
& \delta C_{23}=-\left(\begin{array}{c}
-\sin \gamma \Delta \gamma \sin \vartheta \cos \psi \\
+\cos \gamma \cos \vartheta \Delta \vartheta \cos \psi \\
-\cos \gamma \sin \vartheta \sin \psi \Delta \psi
\end{array}\right) \\
& -(\cos \gamma \Delta \gamma \sin \psi+\sin \gamma \sin \psi \Delta \psi), \\
& \delta C_{31}=\cos \vartheta \Delta \vartheta \text {, } \\
& \delta C_{32}=-\sin \gamma \sin \vartheta \Delta \vartheta+\cos \gamma \Delta \gamma \cos \vartheta \text {, } \\
& \delta C_{33}=-\sin \gamma \Delta \gamma \cos \vartheta-\cos \gamma \sin \vartheta \Delta \vartheta \text {. }
\end{aligned}
$$

The differences of magnetic components in navigation frame can be obtained by transforming (15):

$$
\begin{gathered}
\widetilde{C}_{b}^{n}\left[\begin{array}{l}
m_{b x} \\
m_{b y} \\
m_{b z}
\end{array}\right]-C_{m}^{n}\left[\begin{array}{l}
0 \\
B \\
0
\end{array}\right] \\
=\delta C_{b}^{n}\left[\begin{array}{l}
m_{b x} \\
m_{b y} \\
m_{b z}
\end{array}\right] \\
=\left[\begin{array}{l}
\delta C_{11} m_{b x}+\delta C_{12} m_{b y}+\delta C_{13} m_{b z} \\
\delta C_{21} m_{b x}+\delta C_{22} m_{b y}+\delta C_{23} m_{b z} \\
\delta C_{31} m_{b x}+\delta C_{32} m_{b y}+\delta C_{33} m_{b z}
\end{array}\right] .
\end{gathered}
$$


Attitude errors that are contained in each element in the right side of (18) can be separated from the actual attitude, and the observed equation can be described directly as

$$
Z=H X+V
$$

where $Z$ is observed quantities, $Z=C_{m}^{n}\left[\begin{array}{l}0 \\ B \\ 0\end{array}\right]-C_{b}^{n}\left[\begin{array}{l}m_{b x} \\ m_{b y} \\ m_{b z}\end{array}\right]$, $H$ is measurement matrix, $H=\left[\begin{array}{ccc}H_{1} & H_{2} & H_{3} \\ H_{4} & H_{5} & H_{6} \\ H_{7} & H_{8} & H_{9}\end{array}\right]$, and $V$ is measurement noise, $V=\left[\begin{array}{lll}\zeta_{e} & \zeta_{n} & \zeta_{u}\end{array}\right]^{T}$. In addition, each element of $H$ is formulated as

$$
\begin{aligned}
H_{1}= & (-\cos \gamma \sin \vartheta \sin \psi+\sin \gamma \cos \psi) m_{b y} \\
& +(-\sin \gamma \sin \vartheta \sin \psi+\cos \gamma \cos \psi) m_{b z}, \\
H_{2}= & -\sin \vartheta \sin \psi m_{b x}-\sin \gamma \cos \vartheta \sin \psi m_{b y} \\
& -\cos \gamma \cos \vartheta \sin \psi m_{b z}, \\
H_{3}= & \cos \vartheta \cos \psi m_{b x} \\
& +(-\sin \gamma \sin \vartheta \cos \psi+\cos \gamma \sin \psi) m_{b y} \\
& +(-\cos \gamma \sin \vartheta \cos \psi-\sin \gamma \cos \psi) m_{b z}, \\
H_{4}= & -\cos \gamma \sin \vartheta \sin \psi-\sin \gamma \sin \psi) m_{b y} \\
& +(\sin \gamma \sin \vartheta \cos \psi-\cos \gamma \sin \psi) m_{b z}, \\
H_{5}= & -\sin \vartheta \cos \psi m_{b x}-\sin \gamma \cos \vartheta \cos \psi m_{b y} \\
& -\cos \gamma \cos \vartheta \cos \psi m_{b z}, \\
H_{6}= & -\cos \vartheta \sin \psi m_{b x} \\
& +(-\sin \gamma \sin \vartheta \sin \psi+\cos \gamma \cos \psi) m_{b y} \\
& +(\cos \gamma \sin \vartheta \sin \psi-\sin \gamma \sin \psi) m_{b z}, \\
H_{7}= & \cos \gamma \cos \vartheta m_{b y}-\sin \gamma \cos \vartheta m_{b z}, \\
H_{8}= & \cos \vartheta m_{b x}-\sin \gamma \sin \vartheta m_{b y}-\cos \gamma \sin \vartheta m_{b z}, \\
H_{9}= & 0 .
\end{aligned}
$$

\section{Case Study}

For the sake of verification of the in-flight self-alignment method, we utilize a real experimental data of a spinning projectile whose navigation computer monitored the whole fight data. The flight experiment is made in the northern hemisphere, and the angles of geomagnetic vector are $I=$ $1.00 \mathrm{rad}$ and $D=0.11 \mathrm{rad}$. The total flight time is $65 \mathrm{~s}$, and the interval time is $0.01 \mathrm{~s}$. The trajectory of the spinning projectile is shown in Figure 1, which is obtained by telemetry technology.

The main mind of this process is as follows. We apply the fast coarse alignment method firstly to calculate the initial attitude by using data during $0 s \sim 5$ from Figures 1, 3, and 4 and then apply in-flight alignment model based on Kalman
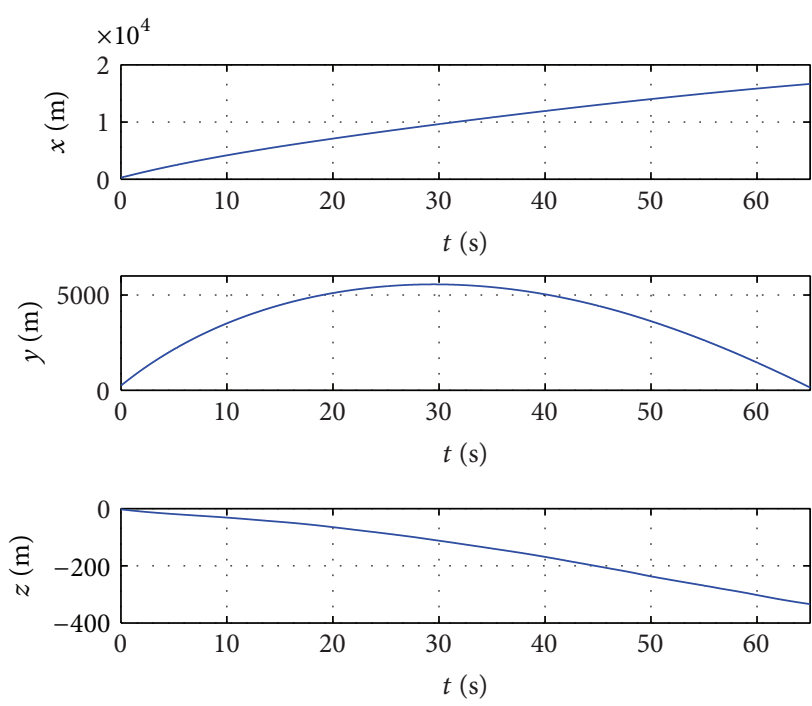

FIGURE 1: Trajectory of the spinning projectile.
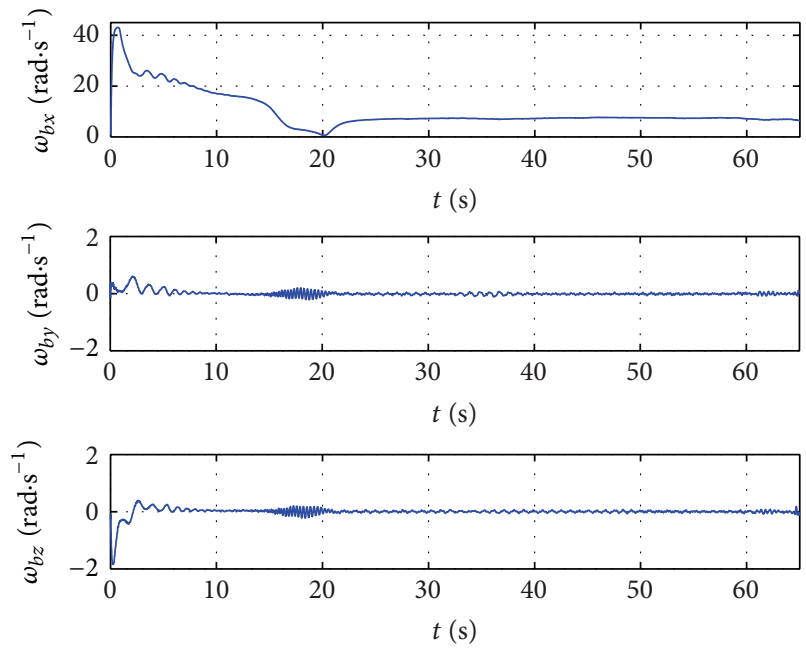

FIGURE 2: Measurement data of the MEMS triaxial gyro.

theory to realize more accurate self-alignment by using data during $5 \mathrm{~s} \sim 65 \mathrm{~s}$.

Figure 2 shows measurement data of the MEMS triaxial gyro fixed in the projectile. A high rolling speed in $x$-axis of the projectile is $43.33 \mathrm{rad} / \mathrm{s}$ at first. Then the rolling speed suffers a fast reduction of speed in front half of the flight, and finally it maintains about $7.35 \mathrm{rad} / \mathrm{s}$ that is still regarded as a high spinning speed.

Figure 3 shows the measurement data of the MEMS triaxial magnetic sensor fixed in the projectile. The longwise component of the sensor is negative during rising stage and then becomes positive during falling stage. The transversal components vary alternately like a sinusoid as the projectile spins. In fact they are the components of projection of geomagnetic vector in body frame during the whole flight.

Figure 4 shows the changing speed of the trajectory angles that can be calculated by using the data of Figure 1. 
TABLE 1: Attitude error by fast coarse method.

\begin{tabular}{lccc}
\hline Time $(\mathrm{s})$ & $\Delta \vartheta(\mathrm{rad})$ & $\Delta \psi(\mathrm{rad})$ & $\Delta \gamma(\mathrm{rad})$ \\
\hline 0.43 & 0.017 & 0.008 & 0.062 \\
0.77 & -0.008 & 0.0014 & 0.051 \\
1.09 & -0.014 & 0.009 & -0.074 \\
2.81 & 0.09 & 0.0041 & 0.081 \\
3.29 & 0.013 & -0.016 & 0.054 \\
4.33 & -0.016 & -0.009 & -0.063 \\
4.67 & 0.026 & 0.012 & 0.049 \\
\hline
\end{tabular}
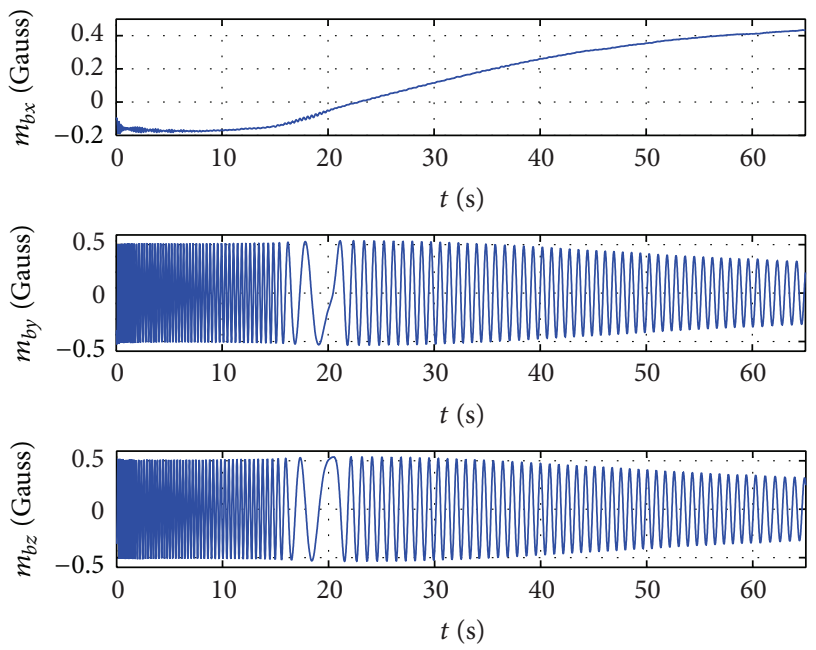

Figure 3: Measurement data of the triaxial magnetic sensor.

From Figure 4, we can see the varying range of $\dot{\theta}$ is $[-0.038 \mathrm{rad} / \mathrm{s}-0.013 \mathrm{rad} / \mathrm{s}]$ and the varying range of $\dot{\psi}$ is $[-0.001 \mathrm{rad} / \mathrm{s}-0.023 \mathrm{rad} / \mathrm{s}]$. They change slowly in the whole time.

Figure 5 provides the attitude by using fast alignment method during $0 s \sim 5 \mathrm{~s}$. It is seen that seven sets are calculated by fast coarse alignment method. It is because not any value of $\vartheta$ by (2) is satisfied, and we choose the value under square root that is less than 1 .

Table 1 shows the attitude error. It is clear that the attitude error is less than five degree and meets the assumption of (8). So the fast coarse alignment method is valid.

We choose the attitude of this projectile at $4.33 \mathrm{~s}$ as the initial attitude that is $\vartheta=0.75 \mathrm{rad}, \psi=-0.00084 \mathrm{rad}$, and $\gamma=0.18 \mathrm{rad}$, and the corresponding actual attitude is $\vartheta=$ $0.72 \mathrm{rad}, \psi=-0.0044 \mathrm{rad}$, and $\gamma=0.13 \mathrm{rad}$.

Based on the coarse initial alignment, we continue to make fine alignment by using the in-flight alignment mode based on Kalman theory. Figure 6 shows the process of the alignment from $5 s \sim 65 s$. It is seen that the attitude error reduces gradually, and the converging speed of rolling angle error is slower than others. When the projectile flies over the peak of the flight, the alignment error has fluctuation near $29 \mathrm{~s}$ due to occurrence of singular of $\vartheta$. Through the alignment
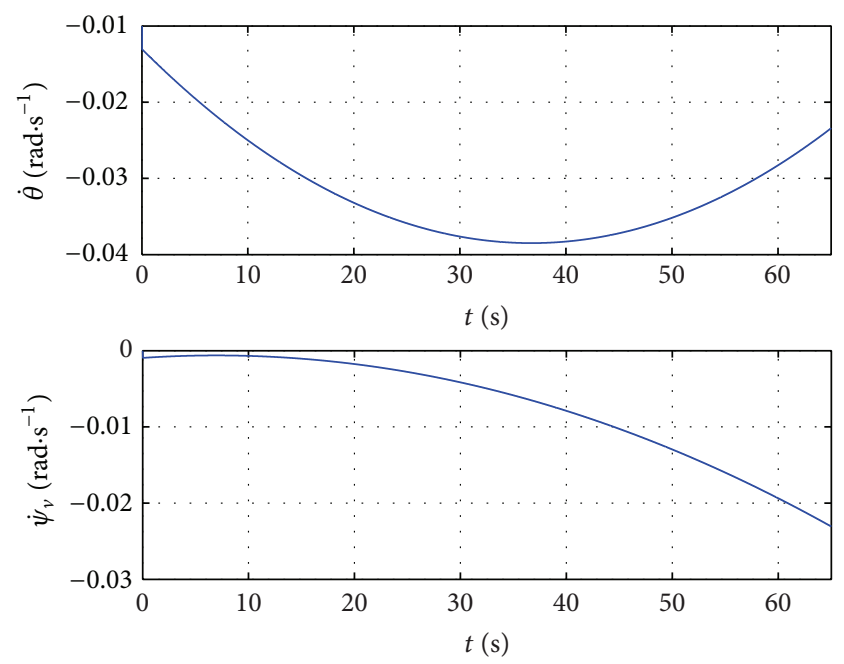

FIGURE 4: The changing rate of the trajectory angles.
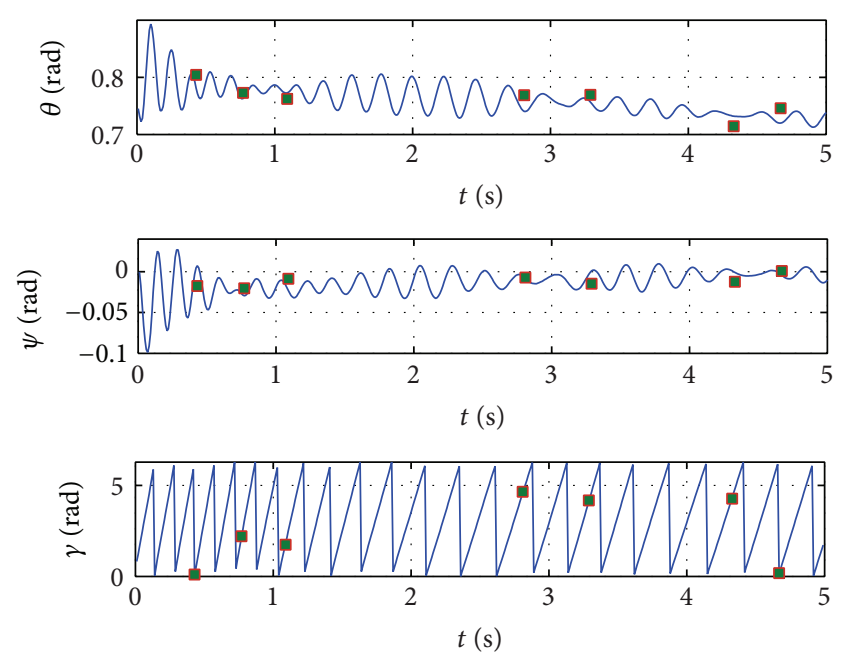

FIGURE 5: Attitude calculated by fast coarse alignment method.
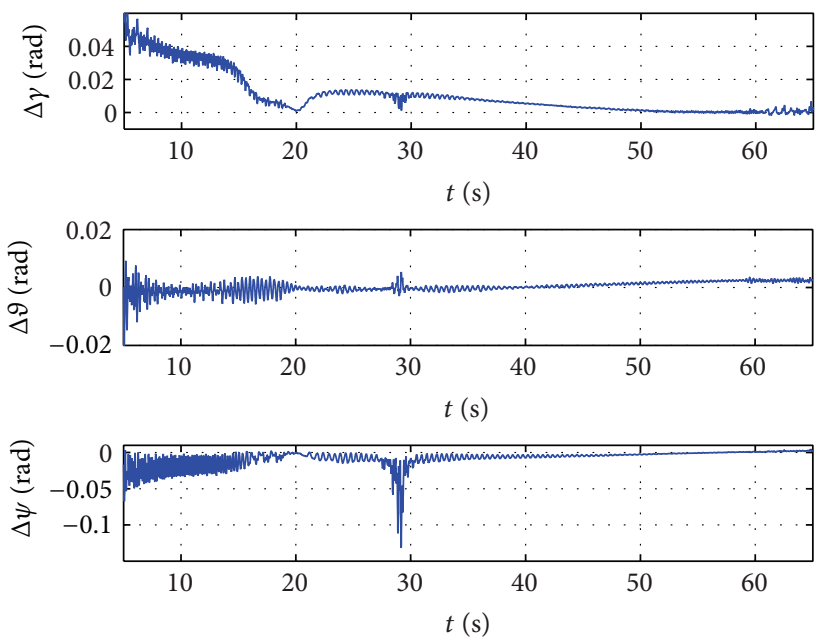

FIgURE 6: The process of the fine alignment by the in-flight alignment mode based on Kalman theory. 

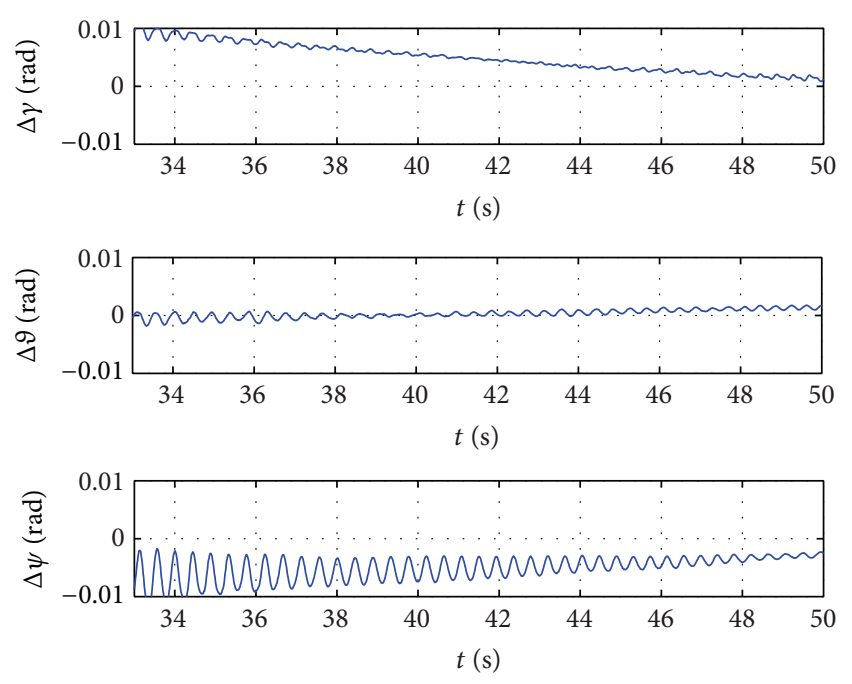

FIGURE 7: Partial magnification of attitude error.

method working, the attitude converges soon. It is apparent that the model has a better adaptability.

Figure 7 shows the partial magnification of attitude error. At $34 \mathrm{~s}$, the attitude error is less than $0.01 \mathrm{rad}$. At $39.26 \mathrm{~s}$, the roll angle error is $0.006 \mathrm{rad}$, pitch angle error is zeros, and yaw angle error is $-0.007 \mathrm{rad}$. It is clear that the maxium error is about $24^{\prime}$, which is high precision level for spining munintions. Although the pitch angle error is increasing after $39.26 \mathrm{~s}$, the final error is $0.0020 \mathrm{rad}$ at $65 \mathrm{~s}$. At the same time, the roll angle error is $0.0067 \mathrm{rad}$, and yaw angle error is $0.0021 \mathrm{rad}$. It is apparent that fine alignment by the in-flight alignment model based on Kalman theory shows satisfying performance, which is achieved finalliy.

\section{Conclusions}

To solve the problem of initial alignment of guided munitions, this paper introduces an in-flight self-alignment method aided by geomagnetism composed of a fast coarse alignment method and an in-flight alignment model by Kalman theory. The fast in-flight coarse alignment method is developed by using gyros, magnetic sensors, and trajectory angle. The in-flight alignment model by Kalman theory is developed through investigation of the measure errors and attitude errors, and this model regards attitude errors as state variables and geomagnetic components in navigation frame as observed variables. Finally, we utilize a data of a spinning projectile to verify the performance of alignment method. The results show that (1) the precision of coarse alignment can attain below $5^{\circ}$; (2) the attitude errors by in-flight alignment model converge to $24^{\prime}$ at early of the latter half of the flight; (3) the in-flight alignment model based on Kalman theory has better adaptability and show satisfying performance. Therefore, the in-flight self-alignment method aided by geomagnetism can meet the requirement of guided munitions with high spinning speed and power-after-launch mode. Furthermore, this method is also suitable for carriers of low rotating speed. In the future, the guidance and control for guided munitions of the high rotating speed will be investigated.

\section{Conflict of Interests}

The authors declare that there is no conflict of interests regarding the publication of this paper.

\section{Acknowledgment}

The grant support from the National Science Foundation of China (nos. 61201417 and 11202023) is greatly acknowledged.

\section{References}

[1] C.-B. Mei, Y.-Y. Qin, and J.-C. You, "SINS in-flight alignment algorithm based on GPS for guided artillery shell," Journal of Chinese Inertial Technology, vol. 22, no. 1, pp. 51-57, 2014.

[2] W.-X. Qian, J.-Y. Liu, R.-B. Li, and Z.-M. Zheng, "Coarse alignment method of airborne SINS in high speed and high dynamic conditions," Journal of Chinese Inertial Technology, vol. 17, no. 4, pp. 388-392, 2007.

[3] M.-J. Yu and S. W. Lee, "A robust extended filter design for SDINS in-flight alignment," International Journal of Control, Automation and Systems, vol. 1, no. 4, pp. 520-526, 2003.

[4] F.-J. Pei, X. Liu, and L. Zhu, "In-flight alignment using $H_{\infty}$ filter for strapdown ins on aircraft," The Scientific World Journal, vol. 2014, Article ID 820305, 8 pages, 2014.

[5] K. K. Veremeenko and V. M. Savel'Ev, "In-flight alignment of a strapdown inertial navigation system of an unmanned aerial vehicle," Journal of Computer and Systems Sciences International, vol. 52, no. 1, pp. 106-116, 2013.

[6] H.-P. She, S.-X. Yang, and H. Ni, "New algorithms to estimate initial roll angle for in-flight alignment of GPS /INS guided munitions," Acta Armamentarii, vol. 32, no. 10, pp. 1265-1270, 2011.

[7] J.-C. Fang and S. Yang, "Study on innovation adaptive EKF for in-flight alignment of airborne POS," IEEE Transactions on Instrumentation and Measurement, vol. 60, no. 4, pp. 1378-1388, 2011.

[8] P. M. G. Silson, “Coarse alignment of a ship's strapdown inertial attitude reference system using velocity loci," IEEE Transactions on Instrumentation and Measurement, vol. 60, no. 6, pp. 19301941, 2011.

[9] A. Acharya, S. Sadhu, and T. K. Ghoshal, "Improved selfalignment scheme for SINS using augmented measurement," Aerospace Science and Technology, vol. 15, no. 2, pp. 125-128, 2011.

[10] L. Ma, K. Wang, and M. Shao, "Initial alignment on moving base using GPS measurements to construct new vectors," Measurement: Journal of the International Measurement Confederation, vol. 46, no. 8, pp. 2405-2410, 2013.

[11] K. Bimal Raj and A. Joshi, "In-motion alignment of inertial navigation system with doppler speed measurements," in Proceedings of the AIAA Guidance, Navigation, and Control Conference, AIAA, Kissimmee, Fla, USA, 2015.

[12] W. Li, K. Tang, L. Lu, and Y. Wu, "Optimization-based INS inmotion alignment approach for underwater vehicles," OptikInternational Journal for Light and Electron Optics, vol. 124, no. 20, pp. 4581-4585, 2013. 
[13] X.-F. Qian, R.-X. Lin, and Y.-N. Zhao, Missile Flight Mechanics, Beijing Institute of Technology Press, Beijing, China, 2000.

[14] G.-X. Shi, S.-X. Yang, and Z. Su, "The study on attitude algorithm of rolling projectile using geomagnetic information," Journal of Projectiles, Rockets, Missiles and Guidance, vol. 31, no. 5, pp. 33-38, 2011. 

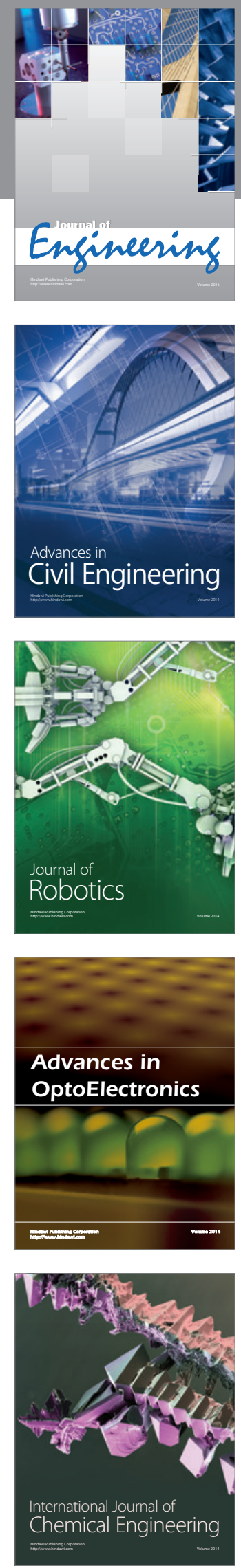

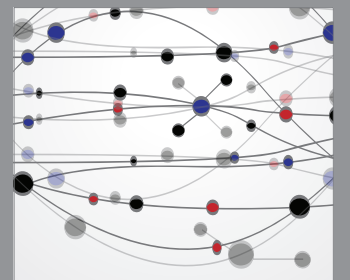

The Scientific World Journal
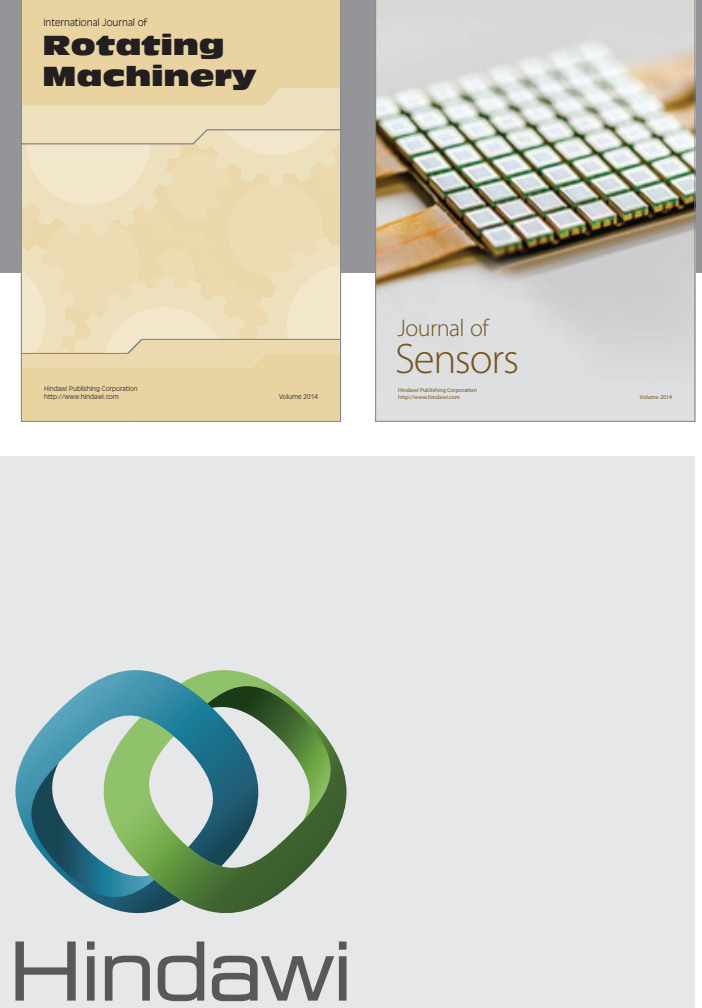

Submit your manuscripts at http://www.hindawi.com
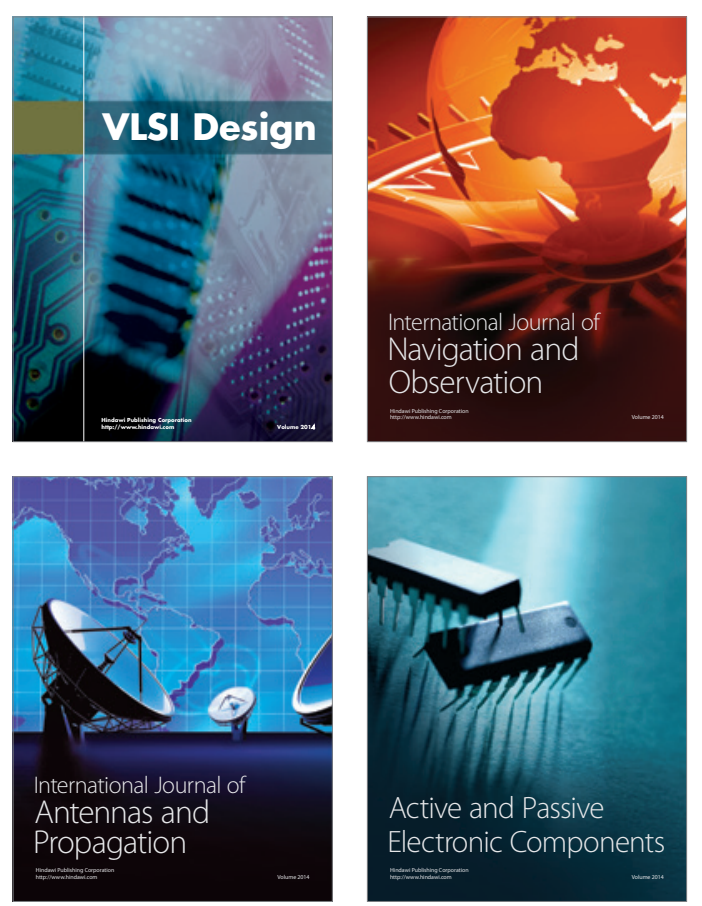
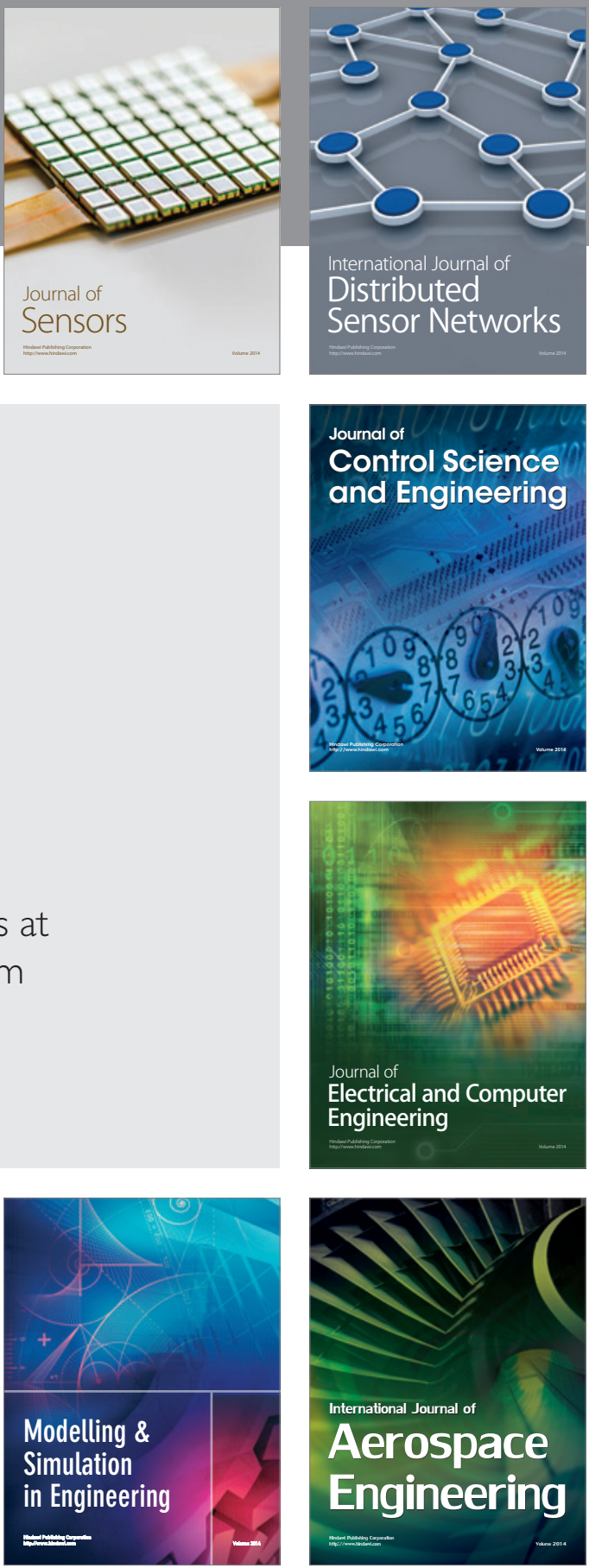

Journal of

Control Science

and Engineering
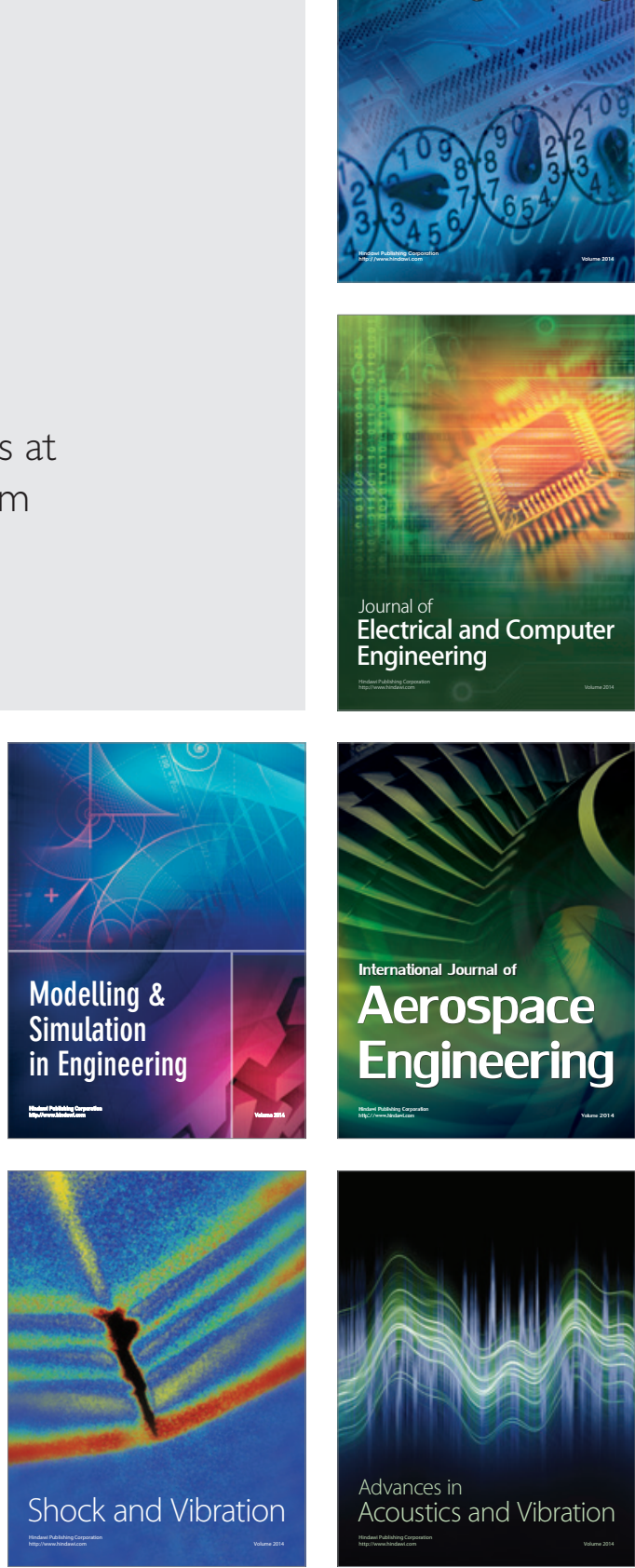